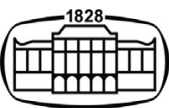

AKADÉMIAI KIADÓ

\section{Journal of Psychedelic} Studies

5 (2021) 1, 37-43

DOI:

10.1556/2054.2021.00165

(c) 2021 The Author(s)

\section{ORIGINAL RESEARCH} PAPER

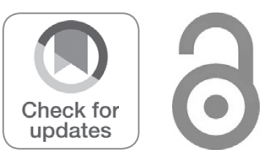

\title{
Symptom specificity of ayahuasca's effect on depressive symptoms
}

\author{
CODY SYKES GILBERT* ${ }^{*}$, MITCH EARLEYWINE, \\ MAHA N. MIAN and BRIANNA R. ALTMAN
}

Department of Psychology, University at Albany, SUNY Albany, NY, 12203, United States

Received: December 20, 2020 • Accepted: March 22, 2021

Published online: April 17, 2021

\begin{abstract}
Background: Ayahuasca's effects on symptoms of depression have generated considerable optimism. Clients frequently report more concern about some symptoms than others, and available treatments alter symptoms differentially. Few studies address the symptom specificity of this psychoactive brew. Aims: We examined self-reported effects of ayahuasca on the individual symptoms of depression assessed by the 10-item short-form of Center for Epidemiological Studies of Depression (CESD-10). Methods: We asked over 120 participants to complete a retrospective assessment of CESD-10 symptoms one month before and one month after using ayahuasca. Results: Participants indicated that ayahuasca had a larger influence on affective symptoms like hope, depressed mood, and happiness, than cognitive, interpersonal, and somatic symptoms like restless sleep, loneliness, and difficulty focusing. Conclusions: Potential clients might appreciate identifying if different treatments provide more relief for some depressive symptoms than others. We examined retrospective reports of ayahuasca's potential for differential impact. Those eager to alter hope, happiness, and other affective symptoms will likely find ayahuasca more helpful than those who want an intervention for restless sleep, loneliness, or trouble focusing. This symptom specificity parallels the effects of serotonergic antidepressant medications, suggesting that psychedelic-assisted psychotherapy using ayahuasca might have considerable appeal for those who seek comparable relief but would rather not use prescription serotonergic medications. Jumpstarting psychotherapy with the rapid onset of ayahuasca-induced relief also appears to have potential.
\end{abstract}

\section{KEYWORDS}

ayahuasca, depression, psychedelic-assisted therapy, symptom specificity, antidepressants

\section{INTRODUCTION}

Major Depressive Disorder is a common, costly, impairing problem, often with chronic and recurring episodes. Many sufferers go undiagnosed (Williams, Chung, \& Muennig, 2017). Global lifetime prevalence estimates have increased nearly $50 \%$ over the last 30 years, and likely exceed 300,000,000 (WHO, 2017). Lifetime prevalence in the United States can be as high as 1 in 5 (Hasin et al., 2018), with associated expenses approaching \$250 billion in today's dollars (Greenberg, Fournier, Sisitsky, Pike, \& Kessler, 2015; US Bureau of Labor Statistics, 2020). Half of those who experience a first episode recover, but at least $35 \%$ report repeated episodes, with $15 \%$ describing the disorder as unremitting (Eaton et al., 2008). Pharmacological and psychological interventions, as well as their combination, show considerable promise but leave many affected individuals unimproved (see Authors, 2020; Cuijpers, Karyotaki, de Wit, \& Ebert, 2020). Pharmacological treatments of choice (Selective Serotonin Reuptake Inhibitors; SSRIs), keep the neurotransmitter in the synapse longer, essentially allowing the post-synaptic neuron to fire repeatedly before serotonin returns to the pre-synaptic neuron for subsequent use (Gabriel et al., 2020). Psychotherapy tends to include candid discussions designed to alter relevant thinking processes and goals in an effort to increase activities consistent with a client's values (Cuijpers et al., 2020). 
Available treatments have serious drawbacks. Antidepressant medications frequently create aversive side-effects, including dry mouth, poor sexual functioning (Read \& Williams, 2018), and increased mortality (Konttinen, Kilpi, Moustgaard, \& Martikainen, 2016). Nearly half (46\%) of those who use these medications report severe withdrawal after discontinuation, which can often last for months (Davies \& Read, 2019). Psychotherapy lacks side effects and withdrawal, but can require considerable time, effort, and expense. Availability is also often limited (See Thase et al., 2020). Efficacy is far from perfect even when health professionals combine both approaches. Some medications help as few as 1 in 9 patients (Citrome, 2016). Standard psychotherapies can have drop-out rates that exceed 50\% (Pentaraki, 2018). Meta-analytic reviews suggest that only $65 \%$ of those in the combined treatment improve (Cuijpers et al., 2020), but the range in success rates is vast. In addition, both pharmacological and psychological interventions rarely create much relief for the initial few weeks (Ramaker \& Dulawa, 2017).

Research investigating which treatments alter which aspects of the disorder might prove beneficial, especially when considering that clients often view some symptoms as more distressing than others. For example, patients frequently report that they are more concerned about returning to work and family life than feeling guiltless or joyful (Eiring et al., 2015; Zimmerman et al., 2006). Standard serotonergic antidepressants also do not address hallmark decreases in pleasure, interest, energy, and positive affect, potentially failing to improve the symptoms a client might find most troublesome (Argyropoulos \& Nutt, 2013; Nutt et al., 2007). A meta-analytic review revealed that medications have small but statistically significant advantages over cognitive behavioral therapy ( $d=0.13-0.16)$ for depressed mood, guilt, suicidal ideation, anxiety, and somatic symptoms but the two treatments are often indistinguishable for other symptoms (Boschloo et al., 2019). (This review did not address pleasure, interest, energy, and positive affect directly, but an item related to "work and activities" did not differ between medication and cognitive behavioral therapy, with differences of only one-ninth of a standard deviation. Perhaps psychotherapy offers no more than medication for these symptoms.) This information might help clinicians and patients choose treatments based upon their own priorities and the intensity of relevant symptoms.

This symptom specificity does raise a problem related to the status of the diagnostic category of clinical depression. Taxometric work reveals that depression lies on a continuum. Depressed people differ from others in degree rather than kind (Haslam, McGrath, Viechtbauer, \& Kuppens, 2020). Delaying interventions until potential clients qualify for a full-blown diagnostic category might lead to missed opportunities at prevention. The idea that interventions could address key aspects of suffering, even for those without a diagnosis, might appeal to potential clients. Depression's dramatic overlap with labels like burnout, exhaustion, and cynicism suggests that many who function worse than they desire might appreciate any of these interventions. For example, formal measures of depressive symptoms and burnout regularly show strong covariation (Koutsimani, Montgomery, \& Georganta, 2019). Research on subtyping of depressive disorders based on clusters of symptoms also has implications for treatment decisions and outcomes (Bianchi, Schonfeld, \& Verkuilen, 2020). Perhaps those who do not qualify for the diagnosis but experience specific symptoms could respond better to one treatment approach than another.

The shortcomings of available treatments related to delayed onset, withdrawal, side effects, and poor efficacy have inspired renewed research in psychedelic-assisted therapy (Romeo, Karila, Martelli, \& Benyamina, 2020). Ayahuasca, a psychoactive plant brew traditionally consumed by native communities in the Amazon Basin (Spruce \& Wallace, 1908), is a combination of the plants Psychotria viridis, a source of the psychedelic N,N-dimethyltryptamine (DMT), with Banisteriopsis caapi, which contains reversible monoamine oxidase inhibitors (Carbonaro et al., 2015; McKenna, Towers, \& Abbott, 1984; Riba, 2003). Laboratory administrations improve depressive symptoms at rates comparable to standard treatments, and with rapid onset of relief (e.g., dos Santos, Sanches, Osório, \& Hallak, 2018; Osorio, 2015; Palhano-Fontes et al., 2019a, 2019b; Palhano-Fontes et al., 2018). A single dose can create clinically meaningful improvements within 24 hours (Palhano-Fontes et al., 2019a, 2019b). This rapid effect of ayahuasca has inspired speculation about jump-starting psychological treatments with the plant medicine. An ideal session (or two) with a relevant psychoactive substance might create initial relief that psychotherapy could enhance and extend (Authors, 2020). Combining ayahuasca treatment with standard psychotherapy might lengthen the period of relief and minimize the chances of relapse, which can reach $50 \%$ over the next two years (Vittengl, Clark, Dunn, \& Jarrett, 2007). This combination approach would not work, however, with most standard pharmacological interventions. Adding ayahuasca to serotonergic medications is ill-advised given the potential for the combination to create "serotonin syndrome," an adverse reaction related to over-activation of the neurotransmitter system (Callaway \& Grob, 1998).

The brew's actions in the serotonin system differ from the impact of standard antidepressants (Carhart-Harris \& Nutt, 2017). Ayahuasca does not share the side effects (Bull et al., 2002; Bultman \& Svarstad, 2002) or potential withdrawal (Read \& Williams, 2018) attributed to the medications. Nevertheless, acute responses are frequently challenging and can include nausea, vomiting, frightening thoughts, delusions, and hallucinations (see Authors, 2020). Fortunately, long-term negative consequences are rare. They often arise from poor pre-morbid functioning or inadequate screening, but hepatic and cardiac problems might arise in those at risk (dos Santos, 2013). Hallucinogen Persisting Perception Disorder, an extremely rare but distressing lingering of the subjective effects, might also be possible (Martinotti et al., 2018). Ayahuasca rarely creates dependence or enduring psychological or physical problems (Barbosa et al., 2018). 
With these caveats in mind, we note that the symptom specificity of ayahuasca's impact has received little attention in empirical work. Identifying specific symptoms targeted by ayahuasca could help potential clients, including those who might not qualify for a formal diagnosis, make informed decisions about standard antidepressant medications, psychotherapy, and ayahuasca. The choice of treatment likely depends upon a delicate balance among the need for rapid relief but also individual availability, expenses, time, and prioritization of symptoms. Thus, the current study compared self-reported pre-post changes for each symptom on the CESD-10 after ayahuasca use. Identifying if ayahuasca's impact on individual symptoms varies from the impact of established pharmacological and psychological interventions could have a meaningful impact, particularly if the brew alters key symptoms in ways that other treatments do not. Perhaps ayahuasca could decrease some symptoms of depression but not others or prevent onset in those experiencing only a few symptoms.

\section{METHODS}

\section{Participants}

Participants responded online via links posted on Facebook pages of groups interested in Ayahuasca. Participants $(N=$ 143) responded anonymously via SurveyMonkey ${ }^{\mathrm{TM}}$ about several demographic variables, and symptoms of depression before and after ayahuasca use. Data were collected in July and August of 2018. All procedures were approved by the local Institutional Review Board. A link to the survey appeared in addition to a brief description of the study, which mentioned ayahuasca but not depression.

\section{Measures}

Demographics. Participants provided age, gender, ethnicity, and level of education. See Table 1.

Lifetime ayahuasca use. Participants answered the question: "Approximately how many times have you used Ayahuasca?" Ayahuasca use ranged from 1 to 1,100 times in life $(M=39.64, S D=135.19$, skew $=6.25)$.

Symptoms of depression. Participants recalled symptoms of depression a month "before" and a month "after" their most recent ceremony, using the ten items from the Center for Epidemiological Sciences Depression Short Form [CES-D10] (Andresen, Malmgren, Carter, \& Patrick, 1994) - a scale with documented convergent and discriminant validity (Authors, 2011). Typical items included, "I felt that everything I did was an effort," and "I felt lonely." The items "I felt hopeful about the future" and "I was happy" were reverse scored. Participants responded on 4-point Likert scales from 1 ("not at all") to 4 ("a great deal"). Cronbach's alpha for the difference between pre and post-depression severity was 0.849 . Change scores ranged from -26.0 to 3.0 ,
Table 1. Demographics

\begin{tabular}{lcc}
\hline Total & & $N=143$ \\
\hline Gender & Female & $70(49.0 \%)$ \\
Age & Male & $73(51.0 \%)$ \\
& $18-20$ & $2(1.4 \%)$ \\
& $21-29$ & $23(16.1 \%)$ \\
& $30-39$ & $36(25.2 \%)$ \\
& $40-49$ & $39(27.3 \%)$ \\
Ethnicity & $50-59$ & $26(18.2 \%)$ \\
& $60+$ & $17(11.9 \%)$ \\
& Caucasian & $124(86.7 \%)$ \\
& Hispanic or Latinx & $7(4.9 \%)$ \\
& Asian & $1(0.7 \%)$ \\
Education & African or Caribbean descent & $0(0.0 \%)$ \\
& Native American or Alaska Native & $1(0.7 \%)$ \\
& Other or mixed & $10(7.0 \%)$ \\
& Some high school & $3(2.1 \%)$ \\
& High school diploma or equivalent & $16(11.2 \%)$ \\
& Some college & $25(17.5 \%)$ \\
& Associate's degree & $10(7.0 \%)$ \\
& Bachelors degree & $42(29.4 \%)$ \\
& Advanced degree & $46(32.2 \%)$ \\
& Missing & $1(0.7 \%)$ \\
\hline
\end{tabular}

Mean $=-9.5, S D=6.2$. CES-D-10 scores prior to Ayahuasca use averaged 17.02 $S D=7.0$, and after use averaged 6.7, $S D=4.7$. Post-test scores are comparable to those that appear in other non-clinical samples (Gonzalez et al., 2017). Two participants had no symptoms at the initial starting point and were omitted from analyses.

\section{RESULTS}

Comparisons among the impact on 10 symptoms required careful attention to avoid differences that might occur by chance, but given how little work appears on symptom specificity, sacrificing power could lead to missed differences that could be potentially meaningful. We used paired t-tests to compare pre-post differences across symptoms, with a family-wise Type I error of $P<0.05$ adjusted using Rom's procedure (Rom, 1990). This approach maintains power without creating spurious findings. $Z$-score transformations suggested that 7 participants had standardized change scores $>3.0$ on at least one symptom; we dropped these participants from analyses. P-P Plots suggested no outliers or meaningful differences from normality-key assumptions for these statistical tests. Details appear in Table 2. Symptoms appear in order of ayahuasca-induced change, with those showing larger ayahuasca-induced impact appearing first.

Ayahuasca had larger effects on affective symptoms like hope, depressed mood, happiness, and fear, but less impact on restless sleep, loneliness, and focus. Change scores were orthogonal to lifetime use of ayahuasca, which we treated as an ordinal variable given the magnitude of its skew. Only the ayahuasca-induced change in the "bothered" item reached statistical significance using a separate family-wise Rom's (1990) correction, and the effect was small (Spearman's rho $=0.17$, 
Table 2. Change in depressive symptoms pre- and post-ayahuasca

\begin{tabular}{|c|c|c|c|c|c|c|c|c|c|c|c|}
\hline \multirow[b]{2}{*}{ CESD symptom } & \multirow[b]{2}{*}{ Mean change } & \multirow[b]{2}{*}{ (SD) change } & \multicolumn{9}{|c|}{ Effect sizes for differences in impact on symptoms } \\
\hline & & & 1 & 2 & 3 & 4 & 5 & 6 & 7 & 8 & 9 \\
\hline 1. Hope & -1.43 & 1.035 & & & & & & & & & \\
\hline 2. Depress & -1.34 & 1.054 & & & & & & & & & \\
\hline 3. Happy & -1.18 & 0.92 & 0.27 & & & & & & & & \\
\hline 4. Fear & -1.08 & 1.06 & 0.30 & & & & & & & & \\
\hline 5. Effort & -1.07 & 1.095 & 0.29 & & & & & & & & \\
\hline 6. Bother & -0.97 & 1.2 & 0.37 & 0.26 & & & & & & & \\
\hline 7. Motive & -0.94 & 1.06 & 0.44 & 0.375 & 0.22 & & & & & & \\
\hline 8. Focus & -0.83 & 1.08 & 0.48 & 0.42 & 0.29 & 0.19 & & & & & \\
\hline 9. Lonely & -0.77 & 1.125 & 0.47 & 0.465 & 0.35 & 0.24 & 0.22 & & & & \\
\hline 10. Sleep & -0.72 & 1.04 & 0.55 & 0.52 & 0.40 & 0.31 & 0.27 & & & & \\
\hline
\end{tabular}

Mean and standard deviations of change in CESD symptoms (from largest to smallest).

Effect sizes (Cohen's $d$ ) only appear when statistically significant.

Blanks cells appear when ayahuasca's impact did not differ across the two symptoms.

Note: Numbers 1-9 in the first row correspond to depressive symptoms in the same order that they appear in the first column.

Hope - I felt hopeful about the future (Reverse scored); Depress - I felt depressed; Happy - I was happy (Reverse scored); Fear - I felt fearful; Effort - I felt that everything I did was an effort; Bother - I was bothered by things that usually don't bother me; Motive - I could not "get going"; Focus - I had trouble keeping my mind on what I was doing; Lonely - I felt lonely; Sleep - My sleep was restless. $P<0.05$.

$P<0.05)$. Gender also only covaried with one of the ayahuasca-induced changes in symptoms. Only the gender effect of change in the "sleep" item reached statistical significance. Men $($ Mean $=-0.91 ; S D=1.005$ reported larger drops in sleep problems than women $($ Mean $=-0.54 ; S D=$ 1.042; $t(138.048)=-2.314, P<0.04, d=0.36)$.

\section{DISCUSSION}

Clinical depression's impact on human suffering is expensive, ubiquitous, and severe. Nearly one in five US citizens will qualify for a diagnosis of Major Depressive Disorder in their lifetimes, and global estimates of prevalence exceed 300 million. Associated losses can include poor quality of life and increased mortality for affected individuals as well as considerable economic burden that often extends beyond affected individuals to their loved ones (Hasin et al., 2018; WHO, 2017). Psychotherapy, standard antidepressant medications, or their combination appear to leave at least $35 \%$ of clients unimproved (see Authors, 2020; Cuijpers, 2020). Some medications alone succeed markedly less often (Citrome, 2016), and some psychotherapies have drop-out rates of 50\% (Pentaraki, 2018). Even medications that improve symptoms often generate uncomfortable side effects and months of withdrawal symptoms (Davies \& Read, 2019; Read \& Williams, 2018). They also have less impact on important symptoms like anhedonia, interest, and energy (Argyropoulos \& Nutt, 2013; Nutt et al., 2007). Psychotherapy can be time-consuming, effortful, pricey, and inaccessible (See Thase et al., 2020; Cuijpers et al., 2020). In addition, both approaches rarely offer relief from symptoms in the first few weeks of treatment (Ramaker \& Dulawa, 2017).

The drawbacks in available therapies inspired clinical trials of psychedelic-assisted treatments (Romeo et al., 2020), including work with ayahuasca, a plant-based medicine originally concocted by natives of the Amazon Basin. Ayahuasca includes sources of N,N-dimethyltryptamine (DMT) and natural MAOI inhibitors. Empirical work confirms that even one administration alleviates relevant symptoms at rates comparable to standard treatments, often with a very rapid onset, few side effects, and no withdrawal (dos Santos et al., 2018; Osorio, et al., 2015; Palhano-Fontes et al., 2019a, 2019b). But the specificity of its impact on individual symptoms is understudied. Available antidepressant medications, and psychotherapy, have a varied effect on different facets of the disorder (Boschloo et al., 2019; Nutt et al., 2007), including complaints common to those who might not qualify for a diagnostic category, like burnout and exhaustion (Koutsimani, et al., 2019). A detailed understanding of ayahuasca's impact could inform treatment decisions. Ideally, clients might choose one or more approaches depending upon their subjective weighting of symptoms, side effects, and other concerns. Ayahuasca's quick relief might also create advantages during the initial stages of psychotherapy. (In contrast, combining ayahuasca with serotonergic medications can create severe adverse reactions; Callaway \& Grob, 1998).

The current sample reported that ayahuasca's impact on affective symptoms (like hopelessness and happiness) exceeded changes in somatic and cognitive symptoms (like sleep disturbances and impaired focus). This impact on positive affect seems unlike standard serotonergic medications, which often do little for positive affect (Nutt et al., 2007). Nevertheless, the smaller improvements on perceived motivation and energy, including items that refer to "effort" and "get(ting) going," seem comparable to the drawbacks also attributed to the serotonergic drugs. An ideal intervention that enhances energy as well as positive affect might 
remain elusive. The current results underscore previous arguments suggesting that medications that activate the norepinephrine and dopamine systems might fill this gap (Nutt et al., 2007). Researchers once praised MDMA, which increases activity in both systems, as a potential antidepressant (Riedlinger \& Riedlinger, 1994). MDMA-assisted treatment for other disorders appear to help depressive symptoms, though those in the placebo group catch up thanks to good psychotherapy (Parrott, 2018). Ketamine, another rapid-acting psychoactive antidepressant, also can alter dopamine function (Beluhon \& Grace, 2014). But both drugs have abuse potential that might give some prescribers pause, particularly for patients with comorbid drug problems (Papaseit et al., 2020).

Although only a huge randomized clinical trial can answer the relevant questions definitively, individuals more concerned about happiness and hopelessness might prefer ayahuasca to standard antidepressants, particularly if rapid onset and minimal side-effects seem critical. Neither approach has cornered the market for those concerned about a lack of energy or motivation. Combining ayahuasca and serotonergic medications seems ill-advised given warnings about "serotonin syndrome," a potentially toxic combination of relevant agonists (Callaway \& Grob, 1998). Nevertheless, ayahuasca might enhance happiness and hope enough to encourage consistent attendance in psychotherapy, which might in turn create novel improvements in energy and motivation after a few sessions (See Authors, 2020). Given the overlap between symptoms of depression and other forms of human suffering, including burnout and distress (Bianchi et al., 2020), relevant treatments might also address concerns in those who might not qualify for a psychiatric diagnosis.

The limitations of the current sample emphasize the need for continued work on ayahuasca's effect on depressive symptoms that includes placebo controls, extensive assessments, huge and diverse samples, and longitudinal followup. Nevertheless, further research on the symptom-specificity of ayahuasca (and other treatments) could benefit many. The current data are limited given their retrospective nature, lack of professional diagnosis or measurement of a major depressive episode, and absence of an enormous, more diverse sample. But the range in efficacy across symptoms suggests that a close examination of the symptom specificity of treatments is worthwhile. Ayahuasca appears to provide as much as half an extra standard deviation in improvement for symptoms like hopelessness and happiness than for focus and sleep.

With these results and their limitations in mind, researchers might consider assessments that include numerous facets and symptoms. The quest for a psychedelic-assisted or alternative treatment for depression could surely benefit. Clinicians and researchers might want to extend psychedelic treatment beyond stereotypical diagnostic categories. Ayahuasca's impact on depressive symptoms might be unlike the impact of standard antidepressants or cognitive behavioral therapy. Perhaps other medications (including other psychedelics) and other therapies have a unique impact on different symptoms. Further work along these lines could help the hundreds of millions of people who struggle with the disorder and its correlates, as well as their friends, family, and colleagues.

\section{REFERENCES}

Andresen, E. M., Malmgren, J. A., Carter, W. B., \& Patrick, D. L. (1994). Screening for depression in well older adults: Evaluation of a short form of the CES-D. American Journal of Preventive Medicine, 10(2), 77-84.

Argyropoulos, S. V., \& Nutt, D. J. (2013). Anhedonia revisited: Is there a role for dopamine-targeting drugs for depression? Journal of Psychopharmacology, 27(10), 869-877.

Authors (2011). Details omitted for anonymous reviewing.

Authors (2020). Details omitted for anonymous reviewing.

Barbosa, P. C. R., Tófoli, L. F., Bogenschutz, M. P., Hoy, R., Berro, L. F., Marinho, E. A. V., et al. (2018). Assessment of alcohol and tobacco use disorders among religious users of ayahuasca. Frontiers in Psychiatry, 9(APR). https://doi.org/10.3389/fpsyt. 2018.00136.

Beluhon, P., \& Grace, A. A. (2014). Restoring mood balance in depression: Ketamine reverses deficit in dopamine-dependent synaptic plasticity. Biological Psychiatry, 76(12), 927-936.

Bianchi, R., Schonfeld, I. S., \& Verkuilen, J. (2020). A five-sample confirmatory factor analytic study of burnout-depression overlap. Journal of Clinical Psychology, 76(4), 801-821.

Boschloo, L., Bekhuis, E., Weitz, E. S., Reijnders, M., DeRubeis, R. J., Dimidjian, S., et al. (2019). The symptom-specific efficacy of antidepressant medication vs. cognitive behavioral therapy in the treatment of depression: Results from an individual patient data meta-analysis. World Psychiatry, 18(2), 183-191.

Bull, S. A., Hu, X. H., Hunkeler, E. M., Lee, J. Y., Ming, E. E., Markson, L. E., et al. (2002). Discontinuation of use and switching of antidepressants: Influence of patient-physician communication. Journal of the American Medical Association, 288(11), 1403-1409. https://doi.org/10.1001/jama.288.11. 1403.

Bultman, D. C., \& Svarstad, B. L. (2002). Effects of pharmacist monitoring on patient satisfaction with antidepressant medication therapy. Journal of the American Pharmaceutical Association, 42(1), 36-43.

Callaway, J. C., \& Grob, C. S. (1998). Ayahuasca preparations and serotonin reuptake inhibitors: A potential combination for severe adverse interactions. Journal of Psychoactive Drugs, 30(4), 367-369.

Carbonaro, T. M., Eshleman, A. J., Forster, M. J., Cheng, K., Rice, K. C., \& Gatch, M. B. (2015). The role of 5-HT2A, 5-HT2C and mGlu2 receptors in the behavioral effects of tryptamine hallucinogens N,N-dimethyltryptamine and N,N-diisopropyltryptamine in rats and mice. Psychopharmacology, 232(1), 275-284. https://doi.org/10.1007/s00213-014-3658-3.

Carhart-Harris, R. L., \& Nutt, D. J. (2017). Serotonin and brain function: A tale of two receptors. Journal of Psychopharmacology, 31(9), 1091-1120. https://doi.org/10.1177/026988111772 5915. 
Citrome, L. (2016). Vortioxetine for major depressive disorder: An indirect comparison with duloxetine, escitalopram, levomilnacipran, sertraline, venlafaxine, and vilazodone, using number needed to treat, number needed to harm, and likelihood to be helped or harmed. Journal of Affective Disorders, 196, 225-233.

Cuijpers, P., Karyotaki, E., de Wit, L., \& Ebert, D. D. (2020). The effects of fifteen evidence-supported therapies for adult depression: A meta-analytic review. Psychotherapy Research, 30(3), 279-293.

Davies, J., \& Read, J. (2019). A systematic review into the incidence, severity and duration of antidepressant withdrawal effects: Are guidelines evidence-based? Addictive Behaviors, 97, 111-121.

Eaton, W. W., Shao, H., Nestadt, G., Lee, B. H., Bienvenu, O. J., \& Zandi, P. (2008). Population-based study of first onset and chronicity in major depressive disorder. Archives of General Psychiatry, 65(5), 513-520.

Eiring, Ø., Landmark, B. F., Aas, E., Salkeld, G., Nylenna, M., \& Nytrøen, K. (2015). What matters to patients? A systematic review of preferences for medication-associated outcomes in mental disorders. BMJ Open, 5(4).

Gabriel, F. C., de Melo, D. O., Fráguas, R., Leite-Santos, N. C., Mantovani da Silva, R. A., et al. (2020). Pharmacological treatment of depression: A systematic review comparing clinical practice guideline recommendations. PLoS one, 15(4), e0231700.

González, P., Nuñez, A., Merz, E., Brintz, C., Weitzman, O., Navas, E. L., et al. (2017). Measurement properties of the Center for Epidemiologic studies depression scale (CES-D 10): Findings from HCHS/SOL. Psychological Assessment, 29(4), 372.

Greenberg, P. E., Fournier, A. A., Sisitsky, T., Pike, C. T., \& Kessler, R. C. (2015). The economic burden of adults with major depressive disorder in the United States (2005 and 2010). The Journal of Clinical Psychiatry, 76(2), 155-162. https://doi.org/ 10.4088/JCP.14m09298.

Hasin, D. S., Sarvet, A. L., Meyers, J. L., Saha, T. D., Ruan, W. J., Stohl, M., et al. (2018). Epidemiology of adult DSM-5 major depressive disorder and its specifiers in the United States. JAMA Psychiatry, 75(4), 336-346.

Haslam, N., McGrath, M. J., Viechtbauer, W., \& Kuppens, P. (2020). Dimensions over categories: A meta-analysis of taxometric research. Psychological Medicine, 1-15.

Konttinen, H., Kilpi, F., Moustgaard, H., \& Martikainen, P. (2016). Socioeconomic position and antidepressant use as predictors of coronary heart disease mortality: A population-based registry study of 362,271 Finns. Psychosomatic Medicine, 78(2), 144-152.

Koutsimani, P., Montgomery, A., \& Georganta, K. (2019). The relationship between burnout, depression, and anxiety: A systematic review and meta-analysis. Frontiers in Psychology, 10, 284.

Martinotti, G., Santacroce, R., Pettorruso, M., Montemitro, C., Spano, M. C., Lorusso, M., et al. (2018). Hallucinogen persisting perception disorder: Etiology, clinical features, and therapeutic perspectives. Brain Sciences, 8(3), 47.

McKenna, D. J., Towers, G. H. N., \& Abbott, F. (1984). Monoamine oxidase inhibitors in South American hallucinogenic plants: Tryptamine and $\beta$-carboline constituents of Ayahuasca. Journal of Ethnopharmacology, 10(2), 195-223. https://doi.org/10.1016/ 0378-8741(84)90003-5.
Nutt, D., Demyttenaere, K., Janka, Z., Aarre, T., Bourin, M., Canonico, P. L., et al. (2007). The other face of depression, reduced positive affect: The role of catecholamines in causation and cure. Journal of Psychopharmacology, 21(5), 461-471.

Osório, F. de L., Sanches, R. F., Macedo, L. R., dos Santos, R. G., Maia-de-Oliveira, J. P., Wichert-Ana, L., et al. (2015). Antidepressant effects of a single dose of ayahuasca in patients with recurrent depression: A preliminary report. Revista Brasileira de Psiquiatria, 37(1), 13-20. https://doi.org/10.1590/1516-44462014-1496.

Palhano-Fontes, F., Barreto, D., Onias, H., Andrade, K. C., Novaes, M. M., Pessoa, J. A., et al. (2018). Rapid antidepressant effects of the psychedelic ayahuasca in treatment-resistant depression: A randomized placebo-controlled trial. Psychological Medicine, 49(4), 1-9. https://doi.org/10.1017/S0033291718001356.

Palhano-Fontes, F., Barreto, D., Onias, H., Andrade, K. C., Novaes, M. M., Pessoa, J. A., et al. (2019a). Rapid antidepressant effects of the psychedelic ayahuasca in treatment-resistant depression: A randomized placebo-controlled trial. Psychological Medicine, 49(4), 1-9. https://doi.org/10.1017/S0033291718001356.

Palhano-Fontes, F., Barreto, D., Onias, H., Andrade, K. C., Novaes, M. M., Pessoa, J. A., et al. (2019b). Rapid antidepressant effects of the psychedelic ayahuasca in treatment-resistant depression: A randomized placebo-controlled trial. Psychological Medicine, 49(4), 655-663.

Papaseit, E., Pérez-Mañá, C., Torrens, M., Farré, A., Poyatos, L., Hladun, O., et al. (2020). MDMA interactions with pharmaceuticals and drugs of abuse. Expert Opinion on Drug Metabolism and Toxicology, 16(5), 357-369.

Parrott, A. C. (2018). MDMA-assisted psychotherapy. The Routledge International Handbook of Psychobiology, 113.

Pentaraki, A. D. (2018). Treatment outcomes in depression: Reducing drop-out rates in cognitive therapy. BJPsych Advances, 24(2), 101-109.

Ramaker, M. J., \& Dulawa, S. C. (2017). Identifying fast-onset antidepressants using rodent models. Molecular Psychiatry, 22(5), 656-665.

Read, J., \& Williams, J. (2018). Adverse effects of antidepressants reported by a large international cohort: Emotional blunting, suicidality, and withdrawal effects. Current Drug Safety, 13(3), 176-186.

Riba, J. (2003). Human pharmacology of ayahuasca: Subjective and cardiovascular effects, monoamine metabolite excretion, and pharmacokinetics. Journal of Pharmacology and Experimental Therapeutics, 306(1), 73-83. https://doi.org/10.1124/jpet.103. 049882.

Riedlinger, T. J., \& Riedlinger, J. E. (1994). Psychedelic and entactogenic drugs in the treatment of depression. Journal of Psychoactive Drugs, 26(1), 41-55.

Rom, D. M. (1990). A sequentially rejective test procedure based on a modified Bonferroni inequality. Biometrika, 77, 663-665.

Romeo, B., Karila, L., Martelli, C., \& Benyamina, A. (2020). Efficacy of psychedelic treatments on depressive symptoms: A meta-analysis. Journal of Psychopharmacology, 34(10), 10791085.

dos Santos, R. G. (2013). A critical evaluation of reports associating ayahuasca with life-threatening adverse reactions. Journal of Psychoactive Drugs, 45(2), 179-188. 
dos Santos, R.G., Sanches, R. F., Osório, F. de L., \& Hallak, J. E. C. (2018). Long-term effects of ayahuasca in patients with recurrent depression: A 5-year qualitative follow-up. Revista de Psiquiatria Clinica, 45(1), 22-24. https://doi.org/10.1590/0101-60830000000149.

Spruce, R., \& Wallace, A. R. (1908). Notes of a botanist on the Amazon \& Andes. Retrieved from https://archive.org/details/ notesofbotanisto11908spru/page/n8.

Thase, M. E., McCrone, P., Barrett, M. S., Eells, T. D., Wisniewski, S. R., Balasubramani, G. K., et al. (2020). Improving costeffectiveness and access to cognitive behavior therapy for depression: Providing remote-ready, computer-assisted psychotherapy in times of crisis and beyond. Psychotherapy and Psychosomatics, 89(5), 307-313.

US Bureau of Labor Statistics. (2020). Available on-line. https:// www.bls.gov/data/inflation_calculator.htm.
Vittengl, J. R., Clark, L. A., Dunn, T. W., \& Jarrett, R. B. (2007). Reducing relapse and recurrence in unipolar depression: A comparative meta-analysis of cognitive-behavioral therapy's effects. Journal of Consulting and Clinical Psychology, 75(3), 475.

Williams, S. Z., Chung, G. S., \& Muennig, P. A. (2017). Undiagnosed depression: A community diagnosis. SSM-population Health, 3, 633-638.

World Health Organization [WHO] (2017). Depression fact sheet. Retrieved from http://www.who.int/mediacentre/factsheets/ fs369/en/.

Zimmerman, M., McGlinchey, J. B., Posternak, M. A., Friedman, M., Attiullah, N., \& Boerescu, D. (2006). How should remission from depression be defined? The depressed patient's perspective. American Journal of Psychiatry, 163(1), 148-150. 